\title{
The Role of Hippocampal Structural Synaptic Plasticity in Repetitive Transcranial Magnetic Stimulation to Improve Cognitive Function in Male SAMP8 Mice
}

\author{
Jiang Ma Jinhua Wang ${ }^{b}$ Chaonan Lv ${ }^{b}$ Jingjuan Pang ${ }^{c}$ Bing Han ${ }^{b}$ Yuan Gen ${ }^{d}$ \\ Mingwei Wang ${ }^{b, d}$ \\ aFaculty of Graduate Studies, Hebei Medical University, Shijiazhuang, 'Department of Neurology, \\ The First Hospital of Hebei Medical University, Shijiazhuang, Institute of Mental Health, North China \\ University of Science and Technology, Tangshan, ${ }^{\mathrm{B} B r a i n}$ Aging and Cognitive Neuroscience Laboratory \\ of Hebei Province, Shijiazhuang, China
}

\section{Key Words}

Synaptic plasticity - Repetitive transcranial magnetic stimulation - Aging - Senescenceaccelerated-prone mouse $8 \cdot$ Cognition $\cdot$ Hippocampus

\begin{abstract}
Background: Repetitive transcranial magnetic stimulation (rTMS) has been used to improve cognitive function, but the stimulation protocols are variable and the underlying mechanism is unclear. Therefore, we intend to examine whether $5 \mathrm{~Hz}$ rTMS with $30 \%$ maximum output could improve cognitive functions in senescence-accelerated-prone mouse 8 (SAMP8) through changing synaptic plasticity. Methods: SAMP8 and senescence-accelerated-prone mouse / resistant 1 (SAMR1) (7-month old male) were randomly divided into 3 groups: SMAP8 rTMS group (P8-rTMS), SMAP8 sham-rTMS group (P8-sham), and SAMR1 sham-rTMS group (R1sham). The P8-rTMS group was treated daily with $5 \mathrm{~Hz}$ rTMS with $30 \%$ maximum output for 14 consecutive days, whereas the other two groups were controls without rTMS stimulation. Morris water maze (MWM) experiment was performed after rTMS or sham treatment to assess the effect of rTMS on cognitive function. Reverse transcription polymerase chain reaction and Western blot assays were used to detect the mRNA and protein expression of presynaptic Synapsin (SYN) and postsynaptic density 95 (PSD95) in the hippocampus of these mice. Results: The mean escape latency of the P8-rTMS group was significantly shorter than that of the P8-sham group. The number of platform crossings of the P8-rTMS group was significantly higher than that of the P8-sham group. rTMS significantly upregulated the protein and mRNA expression of SYN and PSD95 in the hippocampus of p8-rTMS mice compared to those of P8 sham mice. Conclusion: $5 \mathrm{~Hz}$ rTMS with $30 \%$ maximum output enhances learning and memory in the SAMP8 mice. This improvement may be associated with the increased expression of synaptic structure proteins SYN and PSD95 in the hippocampus.




\section{Cellular Physiology Cell Physiol Biochem 2017;41:137-144

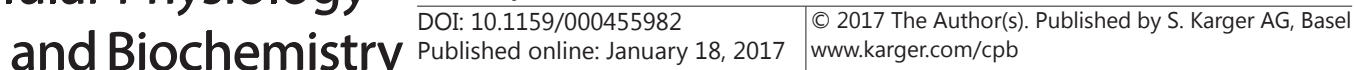 \\ Ma et al.: rTMS Changes Synapsis and Improves Cognitive Function in SAMP8 Mice}

\section{Introduction}

Repetitive transcranial magnetic stimulation (rTMS) is a non-invasive procedure that has been broadly used in treating neurological or psychiatric disorders and been also applied in healthy aging people to improve or maintain cognitive functions [1-3]. Several studies have reported rTMS application in patients with Alzheimer disease (AD), and it has shown to effectively improve their cognitive functions $[4,5]$. However the stimulation protocols are variable and the underlying mechanism is unclear.

To better understand the mechanisms of AD treatment with rTMS, an animal model with phenotypes showing close similarity to those of human is needed. Senescence-acceleratedprone mouse 8 (SAMP8) is a spontaneous animal model that overexpresses amyloid precursor protein and shows all known pathological characteristics of $A D$, including age-associated $A \beta$ deposition in the hippocampus, synaptic dysfunction, and impaired learning and memory $[6,7]$. Compared to other transgenic AD models, SAMP8 has marked dendritic spine loss and late amyloid plaque deposition [7], which are typical in AD patients. In addition, PSD95, a postsynaptic protein, is repressed in SAMP8 mice [8]. Therefore, SAMP8 is an ideal model to study the mechanisms or assess the efficacy of AD treatment with rTMS. Moreover, PSD95 together with SYN could be potential biomarkers for synaptic plasticity.

A common phenotype of AD is learning and memory dysfunction, which is attributed to disrupted synaptic structure and function $[9,10]$. rTMS could alter the structure of dendritic spines and the expressions of synaptic proteins, neurotransmitters, and cognitive related metabolites [11-13]. rTMS could also modulate synaptic transmission causing long-term potentiation or depression (LTP or LTD), which are associated with learning and memory; the neural excitability is determined by the stimulation frequency, where high-frequency increases excitability whereas low frequency reduces excitability $[14,15]$. Other parameters such as stimulus intensity, area of stimulation, current polarity, and stimulation protocol may also alter the synapses differently $[16,17]$.

In our previous studies utilizing aging Kunming mice, we have compared the efficacy of rTMS under different frequencies $(0.5 \mathrm{~Hz}, 1 \mathrm{~Hz}, 5 \mathrm{~Hz}$, and $25 \mathrm{~Hz})$ and intensities $(110 \%$ and $150 \%$ (average resting motor threshold intensity), 20\% and 30\% (maximum output)). We found $5 \mathrm{~Hz}$ rTMS with $30 \%$ maximum output was most effective in improving cognitive functions. The hippocampus is one of the most important brain regions associated with cognition $[18,19]$. Therefore, in the present study, we examined whether $5 \mathrm{~Hz}$ rTMS with $30 \%$ maximum export could reverse the expression of synaptic proteins (PSD95/SYN) in hippocampus and improve learning and memory functions in aging SAMP8 mice.

\section{Materials and Methods}

Animals

SAMP8 and SAMR1 mice provided by Prof. Yew David in the Chinese University of Hong Kong were maintained as an inbred strain. Seven-month-old male SAMP8 mice $(n=40)$ and SAMR1 mice $(n=20)$ weighing $28-32 \mathrm{~g}$ were used in this study. All mice were housed at room temperature $\left(22 \pm 2{ }^{\circ} \mathrm{C}\right)$ in a 12 -h light-dark cycle with food and water ad libitum and adapted to the environment for one week before experiments. The experimental procedures followed the Guidelines for the Care and Use of Animals in Neuroscience Research approved by the Ethnic Committee of Hebei Medical University, China.

The SAMP8 mice were randomly divided into two groups: rTMS group (P8-rTMS group, n=20) and sham-rTMS control group (P8-sham group, $\mathrm{n}=20$ ). The SAMR1 mice were used as a sham-rTMS group (R1sham group, $\mathrm{n}=20$ ). The P8-rTMS group was treated daily with $5 \mathrm{~Hz}$ rTMS for 14 consecutive days whereas the P8-sham group and R1-sham group were handled in a manner similar to that of the rTMS group but without rTMS stimulation. Mice were awake during the stimulation delivery.

Ten mice from each group were randomly selected for RT-PCR and Western blot analysis, and the remaining ten mice were used for the MWM tests. 


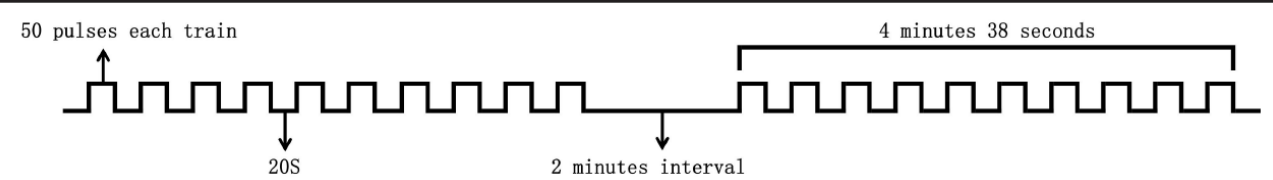

Fig. 1. rTMS protocol delivered in each day. Two sessions of rTMS with 1000 pulses in 20 trains are delivered on 14 consecutive days. The time interval between the two sessions is 2 minutes to cool down the coil; the time interval between each train was 20 seconds. Thus each rTMS session lasts for 4 minutes and 38 seconds.

\section{Application of rTMS}

The procedure of rTMS was performed on the basis of previous clinical and animal studies [20-23]. Briefly, rTMS was delivered with an MC-B70 butterfly coil (MagVenture, Farum, Denmark; inner diameter $20 \mathrm{~mm}$, outer diameter $100 \mathrm{~mm}$ ) connected to a MagProX100 magnetic stimulation device (MagVenture; active pulse width $280 \mu \mathrm{s}, 4.2$ Tesla maximum output) [12]. The p8-rTMS group was exposed to medium frequency rTMS $(5 \mathrm{~Hz})$, with magnetic stimulation intensity set at $30 \%$ maximum output (1.26T). Two sessions of rTMS consisting of 1000 pulses in 10 trains were delivered for 14 consecutive days. There was an interval of 2 minutes between the two sessions to cool down the coil. The interval between each train was 20 second, thus each rTMS session lasted for 4 minutes and 38 seconds (Fig. 1). The sham group mice were treated similarly to the rTMS group by the reverse side of the coil, but were separated from the head using a $3 \mathrm{~cm}$ plastic spacer cube [12]. This ensured that the animal felt the vibrations produced by the click of the rTMS coil without brain stimulation. None of the mice died during the application of stimulation. All rTMS protocols were administered between 14:30 and 17:00.

Since a previous study indicated that the lasting neurochemical outcomes of rTMS treatment might be crucially affected by the conscious state (i.e., anesthesia) during the stimulation [13], we kept the mice awake during the experiment. In order to reduce stress response, we created a plastic cylindrical mold that can be held by hands to make the mice cooperate during the rTMS process. Before rTMS administration, the mice were put into this mold, whose mouth is narrower than the back part and can just keep the mice restricted with its head out. Thus the mice could stay in this cubbyhole comfortably with minimal stress when the stimulation was carried out and the 8-shaped coil was placed above the head of the animal. The longitudinal axis of the coil was perpendicular to the midline and the center of the coil was over the bregma region. The mice were kept with the mold for three days before experiments.

\section{MWM test}

One day after the 14-day magnetic stimulation, ten mice from each group were tested in MWM (between 14:30 and 17:00) to assess spatial learning and memory functions as previously described but with slight modifications $[12,13,24]$. A circular pool $(120 \mathrm{~cm}$ in diameter) was filled with water $(45 \mathrm{~cm}$ in depth) maintained at $20-22^{\circ} \mathrm{C}$; a platform was set up approximately $1 \mathrm{~cm}$ under the water surface and 4 geometric designs at the edge of the pool were served as distal searching cues in each quadrant. Nontoxic black ink was added into the water to make the platform invisible. The mouse was gently put in one of the quadrants in the pool, with its head facing the wall. Each mouse was given 60 seconds to find the platform and the time used to find the platform was recorded as the escape latency. If a mouse failed to locate the platform within 60 seconds, it was guided to the platform, where it stayed for 10 seconds, and the escape latency was considered as 60 seconds. Every mouse had 4 chances to swim each day and was put in the water of the pool at the first, second, third, fourth quadrant sequentially. This swimming test was repeated for five continues days. At day 6, the platform was taken away and each mouse was released at the first quadrant and allowed to swim for 60 seconds in the pool. To measure the platform location retention, each mouse crossing the previous platform location was observed and the number of times was recorded. All performances were recorded by the ANY-maze Behavior Analysis System, with escaping latency and number of crossings as parameters.

\section{Western blot assay}

After decapitation, the brain tissue of the mice was collected and snap-frozen. The hippocampus was then dissected and incubated on ice 20 minutes with radio immunoprecipitation assay lysis buffer containing $1 \%$ phenylmethanesulfonyl fluoride. After homogenizing with a glass homogenizer, samples 


\section{Cellular Physiology Cell Physiol Biochem 2017;41:137-144 \begin{tabular}{ll|l} 
DOI: 10.1159/000455982 & $\begin{array}{l}\text { O 2017 The Author(s). Published by S. Karger AG, Basel } \\
\text { www.karger.com/cpb }\end{array}$ \\
\cline { 2 - 3 }
\end{tabular} \\ Ma et al.: rTMS Changes Synapsis and Improves Cognitive Function in SAMP8 Mice}

were centrifuged at $12,000 \times \mathrm{g}$ for 30 minutes and the supernatant was collected. The bicinchoninic acid assay kit (Sigma-Aldrich, USA) was used to measure the protein concentration.

Next, $50 \mu \mathrm{g}$ samples were subjected to SDS-PAGE (sodium dodecyl sulfate polyacrylamide gel electrophoresis) and then transferred onto polyvinylidene fluoride membranes (Millipore, Bedford, MA, USA). After blocking with $5 \%$ non-fat milk for $45 \mathrm{~min}$ at room temperature, the membranes were incubated with the indicated primary antibodies at $4{ }^{\circ} \mathrm{C}$ overnight with head-over-tail rotation. The membranes were then washed with TBST three times and incubated with HRP-conjugated secondary antibodies (Abcam) for $1 \mathrm{hr}$ at room temperature and then developed with enhanced chemiluminescence kit (ECL, Millipore). The following primary antibodies (Abcam, Cambridge, MA, USA) were used: rabbit anti-SYN (1:10,000 dilution), PSD95 $(1: 2,000)$ and rabbit anti-beta-Actin $(1: 2,000)$. ChemiDoc XRS system and Quantity one software (BIO-RAD, USA) were then used to digitize the band density. The levels of SYN and PSD95 proteins were normalized against the corresponding beta-Actin control protein and then expressed as a percentage of the control samples (R1-sham group, defined as 100\%).

Semi-quantitative reverse transcription PCR (semi-RT-PCR) assay

Qiagen Rneasy mini kit (74014) was used to isolate total RNA from the hippocampus tissue. Then, primescript RT kit (Takara, Japan) and PCR ExTaq (Takara) were used to perform reverse transcription PCR according to the manufacturer's protocol. The amplified PCR products were then resolved by electrophoresis on a $1.5 \%$ agarose gel and analyzed by Quantity one software (Bio-Rad). The expression levels of SYN and PSD95 mRNA were normalized to beta-Actin control and then expressed as a percentage of the control samples (R1-sham group, defined as 100\%). The primer sequences were:

SYN:5'-TTTGCCATCTTCGCCTTTGC-3'(forward);

5'-CACCCGAGGAGGAGTAGTCA-3'(reverse); PSD95:5'-CCCCCAACATGGACTGTCTC-3' (forward); 5'-TGTTCCGTTCACCTGCAAC-3'(reverse); beta-Actin:5'GGTCAGAAGGATTCCTATGTG-3'(forward); 5'-ATTGCCAATGGTGATGACCTG-3' (reverse).

\section{Data analysis}

All data were presented as mean \pm standard deviation (SD). Differences in the place navigation test in MWM were detected using repeated measures ANOVA followed by the least significant difference (LSD) post-hoc analysis. All other experiments were examined by one-way ANOVA. All statistical analyses were performed using SPSS 19.0, and the statistical significance was defined as $\mathrm{P}<0.05$.

\section{Results}

rTMS ameliorates cognitive deficit in SAMP8 mice

To examine the effects of rTMS on learning and memory in SAMP8 mice, we used classic MWM and compared the results among P8-rTMS, P8-sham, and R1-sham mice. The mean escape latency of each mouse finding the platform from the four quarters was recorded in each of the five days. As usual practice, the mean escape latency of the mice became shorter with time during the course of the trials. The mean escape latency of P8-rTMS group was significantly shorter than that of P8-sham group (Fig. 2A, $P=0.022<0.05$ ). The escape latency on these days was markedly shorter in R1-sham group than those in P8-rTMS group or P8sham group (Fig. $2 \mathrm{~A}, P<0.01$ ). Differences among groups were clearly shown at day 6 (Fig. 2B, R1-sham Vs P8-sham or P8-rTMS, $P<0.01$, and P8-rTMS Vs P8-sham, $P=0.019<0.05$ ). The number of platform crossings was markedly higher in R1-sham group than those in P8-rTMS group or P8-sham group (Fig. 2C, $P<0.01$ ). The number of platform crossings for the P8rTMS group was significantly (Fig. $2 \mathrm{C}, P<0.01$ ) higher than those for P8-sham groups. These results suggest that 14 days of rTMS could significantly reverse the impairments of learning and memory in SAMP8 mice, but could not recover to normal levels as in SAMR1 mice.

rTMS upregulates protein expression of SYN and PSD95 in SAMP8 mice

The hippocampus is an important component of the brain in learning and memory. PSD95 and SYN proteins represent two important biomarkers for synaptic plasticity [25, 26]. To evaluate the effect of rTMS on synaptic plasticity, we examined the expressions of PSD95 


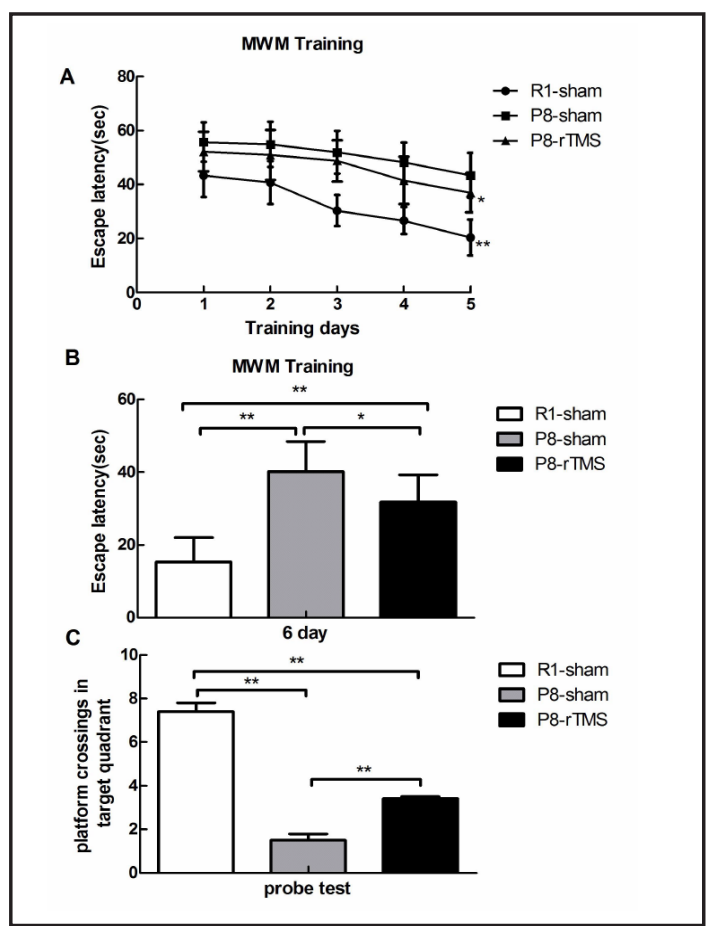

Fig. 2. rTMS ameliorates cognitive deficit in SAMP8 mice by MWM test. The results of MWM tests in P8-rTMS, P8-sham, and R1-sham mice are displayed in graphs showing escape latency on each training day (A), escape latency on the last day (B), and number of platform crossings to the target quadrant during probe test (over 60 seconds) (C). The data are expressed as mean \pm SD $\left(n=10\right.$ /group). ${ }^{*} P<0.05$, ${ }^{*} P<0.01$. Differences in the place navigation test in MWM were detected using repeated measures ANOVA followed by the least significant difference (LSD) post-hoc analysis (data in Fig. 2A). All other experiments were examined by one-way ANOVA.

Fig. 4. rTMS upregulates the mRNA expression of SYN and PSD95 in SAMP8 mice. The expressions of SYN and PSD95 in the hippocampus of P8-rTMS, P8-sham, and R1-sham mice are shown by RT-PCR (A) and the quantification graphs are displayed (B). $\beta$-actin was included as a loading control. The results are expressed as mean \pm SD $(n=5)$. Statistical analysis was performed by using one-way ANOVA with LSD post-hoc test. ${ }^{*} P<0.01$. M: Marker.

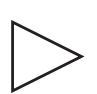

A

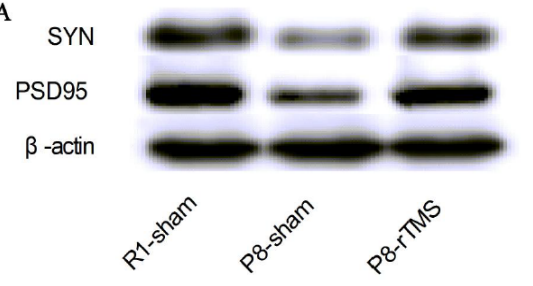

B

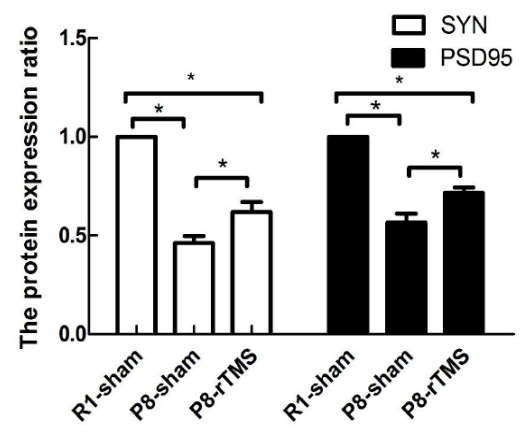

Protein expressions of SYN and PSD95

Fig. 3. rTMS upregulates the protein expression of SYN and PSD95 in SAMP8 mice. Protein expressions of SYN and PSD95 in the hippocampus of P8-rTMS, P8-sham, and R1-sham mice are shown by Western blots (A) and the quantification graph (B). The results are expressed as mean \pm SD $(n=5)$, One-way ANOVA followed by Fisher's LSD post hoc. ${ }^{*} P<0.01$.
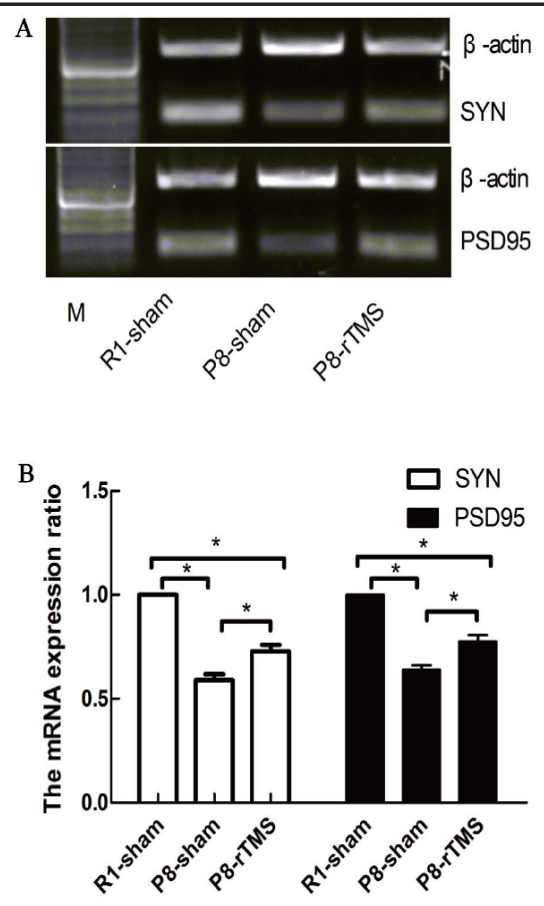

mRNA expressions of SYN and PSD95 


\section{Cellular Physiology Cell Physiol Biochem 2017;41:137-144

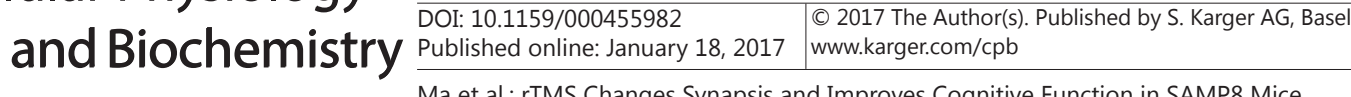

and SYN in the hippocampus of P8-rTMS, P8-sham, and R1-sham mice at protein level. Our results showed that 14 days of rTMS significantly upregulated the protein expression of SYN and PSD95 in the hippocampus of p8-rTMS mice compared to those in P8-sham mice (Fig. 3, $\mathrm{P}<0.01$ ), yet it was lower than the normal level shown in R1-sham control mice.

\section{rTMS upregulates mRNA expression of SYN and PSD95 in SAMP8 mice}

To examine whether the alteration in the expression levels of PSD95 and SYN proteins was due to changes that happen at mRNA level, we performed RT-PCR on hippocampal samples collected from P8-rTMS, P8-sham, and R1-sham mice. The mRNA expression levels of SYN and PSD95 in the different groups were consistent with those of the corresponding proteins (Fig. 4). In addition, the mRNA levels of SYN and PSD95 were the highest in the R1-sham group, significantly higher than those in the P8-Sham group and P8-rTMS group $(P<0.01)$. Results from the semi-RT-PCR and relative mRNA expression analyses (Fig. 4B) revealed that rTMS significantly upregulated the mRNA expression of SYN and PSD95 in p8rTMS mice compared to those in the P8-sham mice $(P<0.01)$.

\section{Discussion}

$\mathrm{AD}$ is a neurological disorder strongly associated with aging and lacks an effective treatment. Recently, rTMS has been applied in aging and AD patients to improve their cognitive functions $[4,5]$. Based on previous work in our lab, we examined the effect of rTMS at $5 \mathrm{~Hz}$ with $30 \%$ maximum output in SAMP8 aging mice. To our knowledge, this is the first rTMS study in SAMP8 mice, an ideal animal model for AD. Here, we proved that 14-day rTMS following our protocol could ameliorate the learning and memory dysfunction in SAMP8 mice, which is also accompanied by upregulation of SYN/PSD95 proteins in the hippocampus. This preliminary study provides remarkable insights to further explore the underlying mechanisms of rTMS in treating aging and AD and clarify the contradictory results from different stimulation protocols.

In a closely related study using transgenic AD mice, rTMS was applied at 1, 10 or 15 $\mathrm{Hz}$ daily for 4 weeks, it frequency-dependently increased the expression of Homer1a (a postsynaptic scaffold protein), enhanced LTP, and ameliorated spatial learning deficits in these mice [27]. In a similar study in normal mice, $15 \mathrm{~Hz}$ rTMS was able to enhance LTP and improve memory but 1 and 8Hz rTMS impaired memory [28]. This discrepancy may be attributed to different protocols used for rTMS in addition to other parameters (intensity, area of stimulation) that may play a role. In an rTMS study in aging mice, low intensity stimulation increased the expression of synaptic proteins whereas high intensity stimulation did the opposite $[12,23]$. A study in our lab also supports that rTMS at low to medium frequency and intensity is beneficial whereas that at high frequency and intensity is detrimental (reported in another paper submitted). Therefore, the rTMS protocol used in the current study could be a starting point to study other parameters in the application of rTMS in SAMP8 model.

A common model of rTMS in improving cognitive function could be summarized from several studies focusing on synaptic plasticity. rTMS at appropriate frequency, intensity, and duration may alter the expression of neurotrophins and their receptors $[12,23,29$, $30]$, neurotransmitters and their receptors [14, 30-32], and structural proteins [12, 23] in synapses, which in turn affects the postsynaptic depolarization, inducing LTP or LTD and contributing to different cognitive functions.

In the present study, we observed the SYN/PSD95 upregulation in rTMS treated SMAP8 mice after 14 days of stimulation at a medium frequency and low intensity and this alteration of synaptic biomarkers is paralleled with the improvement of cognitive function in these mice. This finding suggests the association between synaptic protein levels and cognitive functions where rTMS may modulate cognitive function through altering synaptic plasticity, as proposed by other studies $[12,23]$. Although this result is consistent with other reports, there are some limitations that should be considered in this study. First, due to experimental limitation, we could not identify the specific types of synapses in the hippocampus, such as

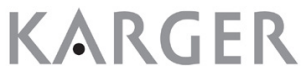




\section{Cellular Physiology Cell Physiol Biochem 2017;41:137-144

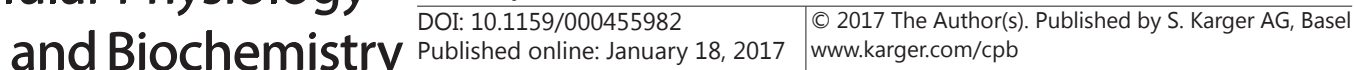 \\ Ma et al.: rTMS Changes Synapsis and Improves Cognitive Function in SAMP8 Mice}

excitatory or inhibitory, glutamatergic or GABAergic, dendritic or somatic, granular neurons or pyramidal neurons, though the total levels for SYN/PSD95 are increased. Second, rTMS may dynamically alter the expressions of synaptic proteins and change the spine size/density in different synapses [11]; thus we could not deduce the long-term effects of rTMS beyond 14 days. Third, rTMS studies in human cases suggest that different area of stimulation may cause opposite results [14]. The stimulation protocol in the present study is adopted from non-specific stimulation models with the coil covering all the top areas of the brain; thus we cannot tell if this effect is restricted to the hippocampus.

In summary, we created a new mouse model to study rTMS application in AD on the basis of previous work in aging mice. In the future, we will continue to dissect the synaptic plasticity after rTMS in SAMP8 mice more precisely at cellular and molecular levels, and examine different areas of stimulation to make the rTMS more focusing and controllable.

\section{Acknowledgements}

This work was Supported by Provincial Major Medical Programs (ZD2013077, ZD2013079) and Hebei Health and Family Planning Commission Directive Program (ZL20140184).

\section{Disclosure Statement}

The authors declare no conflicts of interest.

\section{References}

1 Ljubisavljevic MR, Ismail FY, Filipovic S: Transcranial magnetic stimulation of degenerating brain: a comparison of normal aging, Alzheimer's, Parkinson's and Huntington's disease. Curr Alzheimer Res 2013;10:578-596.

-2 Downar J, Blumberger DM, Daskalakis ZJ: The Neural Crossroads of Psychiatric Illness: An Emerging Target for Brain Stimulation. Trends Cogn Sci 2016;20:107-120.

-3 Tatti E, Rossi S, Innocenti I, Rossi A, Santarnecchi E: Non-invasive brain stimulation of the aging brain: State of the art and future perspectives. Ageing Res Rev 2016;29:66-89.

4 Ahmed MA, Darwish ES, Khedr EM, El Serogy YM, Ali AM: Effects of low versus high frequencies of repetitive transcranial magnetic stimulation on cognitive function and cortical excitability in Alzheimer's dementia. J Neurol 2012;259:83-92.

5 Liao X, Li G, Wang A, Liu T, Feng S, Guo Z, Tang Q, Jin Y, Xing G, McClure MA, Chen H, He B, Liu H, Mu Q: Repetitive Transcranial Magnetic Stimulation as an Alternative Therapy for Cognitive Impairment in Alzheimer's Disease: A Meta-Analysis. J Alzheimers Dis 2015;48:463-472.

6 Yagi H, Katoh S, Akiguchi I, Takeda T: Age-related deterioration of ability of acquisition in memory and learning in senescence accelerated mouse: SAM-P/8 as an animal model of disturbances in recent memory. Brain Res 1988;474:86-93.

7 Morley JE, Armbrecht HJ, Farr SA, Kumar VB: The senescence accelerated mouse (SAMP8) as a model for oxidative stress and Alzheimer's disease. Biochim Biophys Acta 2012;1822:650-656.

-8 Li Q Zhao HF, Zhang ZF, Liu ZG, Pei XR, Wang JB, Li Y: Long-term green tea catechin administration prevents spatial learning and memory impairment in senescence-accelerated mouse prone- 8 mice by decreasing Abeta1-42 oligomers and upregulating synaptic plasticity-related proteins in the hippocampus. Neuroscience 2009;163:741-749.

-9 van der Zee EA: Synapses, spines and kinases in mammalian learning and memory, and the impact of aging. Neurosci Biobehav Rev 2015;50:77-85.

10 Oberman L, Pascual-Leone A: Changes in plasticity across the lifespan: cause of disease and target for intervention. Prog Brain Res 2013;207:91-120.

11 Vlachos A, Muller-Dahlhaus F, Rosskopp J, Lenz M, Ziemann U, Deller T: Repetitive magnetic stimulation induces functional and structural plasticity of excitatory postsynapses in mouse organotypic hippocampal slice cultures. J Neurosci 2012;32:17514-17523. 


\section{Cellular Physiology Cell Physiol Biochem 2017;41:137-144 \begin{tabular}{ll|l} 
and Biochemistry 10.1159/000455982 & $\begin{array}{l}\text { C } 2017 \text { The Author(s). Published by S. Karger AG, Basel } \\
\text { www.karger.com/cpb }\end{array}$ \\
\hline
\end{tabular} \\ Ma et al.: rTMS Changes Synapsis and Improves Cognitive Function in SAMP8 Mice}

12 Ma J, Zhang Z, Kang L, Geng D, Wang Y, Wang M, Cui H: Repetitive transcranial magnetic stimulation (rTMS) influences spatial cognition and modulates hippocampal structural synaptic plasticity in aging mice. Exp Gerontol 2014;58:256-268.

13 Wang H, Geng Y, Han B, Qiang J, Li X, Sun M, Wang Q, Wang M: Repetitive transcranial magnetic stimulation applications normalized prefrontal dysfunctions and cognitive-related metabolic profiling in aged mice. PLoS One 2013;8:e81482.

14 Huang YZ, Rothwell JC, Chen RS, Lu CS, Chuang WL: The theoretical model of theta burst form of repetitive transcranial magnetic stimulation. Clin Neurophysiol 2011;122:1011-1018.

-15 Torii T, Sato A, Nakahara Y, Iwahashi M, Itoh Y, Iramina K: Frequency-dependent effects of repetitive transcranial magnetic stimulation on the human brain. Neuroreport 2012;23:1065-1070.

16 Pilato F, Profice P, Ranieri F, Capone F, Di Iorio R, Florio L, Di Lazzaro V: Synaptic plasticity in neurodegenerative diseases evaluated and modulated by in vivo neurophysiological techniques. Mol Neurobiol 2012;46:563-571.

17 Koch G: Repetitive transcranial magnetic stimulation: a tool for human cerebellar plasticity. Funct Neurol 2010;25:159-163.

18 Wei R, Zhang R, Xie Y, Shen L, Chen F: Hydrogen Suppresses Hypoxia/Reoxygenation-Induced Cell Death in Hippocampal Neurons Through Reducing Oxidative Stress. Cell Physiol Biochem 2015;36:585-598.

19 Yan J, Huang Y, Lu Y, Chen J, Jiang H: Repeated administration of ketamine can induce hippocampal neurodegeneration and long-term cognitive impairment via the ROS/HIF-1alpha pathway in developing rats. Cell Physiol Biochem 2014;33:1715-1732.

20 Piccirillo JF, Kallogjeri D, Nicklaus J, Wineland A, Spitznagel EL, Jr., Vlassenko AG, Benzinger T, Mathews J, Garcia KS: Low-frequency repetitive transcranial magnetic stimulation to the temporoparietal junction for tinnitus: four-week stimulation trial. JAMA Otolaryngol Head Neck Surg 2013;139:388-395.

-21 Gersner R, Kravetz E, Feil J, Pell G, Zangen A: Long-term effects of repetitive transcranial magnetic stimulation on markers for neuroplasticity: differential outcomes in anesthetized and awake animals. J Neurosci 2011;31:7521-7526.

-22 Tan T, Xie J, Liu T, Chen X, Zheng X, Tong Z, Tian X: Low-frequency (1 Hz) repetitive transcranial magnetic stimulation (rTMS) reverses Abeta(1-42)-mediated memory deficits in rats. Exp Gerontol 2013;48:786794.

23 Ma J, Zhang Z, Su Y, Kang L, Geng D, Wang Y, Luan F, Wang M, Cui H: Magnetic stimulation modulates structural synaptic plasticity and regulates BDNF-TrkB signal pathway in cultured hippocampal neurons. Neurochem Int 2013;62:84-91.

24 Ma Q, Qiang J, Gu P, Wang Y, Geng Y, Wang M: Age-related autophagy alterations in the brain of senescence accelerated mouse prone 8 (SAMP8) mice. Exp Gerontol 2011;46:533-541.

25 Castejon OJ, Fuller L, Dailey ME: Localization of synapsin-I and PSD-95 in developing postnatal rat cerebellar cortex. Brain Res Dev Brain Res 2004;151:25-32.

26 Wang J, Yuan J, Pang J, Ma J, Han B, Geng Y, Shen L, Wang H, Ma Q, Wang Y, Wang M: Effects of Chronic Stress on Cognition in Male SAMP8 Mice. Cell Physiol Biochem 2016;39:1078-1086.

-27 Wang F, Zhang Y, Wang L, Sun P, Luo X, Ishigaki Y, Sugai T, Yamamoto R, Kato N: Improvement of spatial learning by facilitating large-conductance calcium-activated potassium channel with transcranial magnetic stimulation in Alzheimer's disease model mice. Neuropharmacology 2015;97:210-219.

-28 Ahmed Z, Wieraszko A: Modulation of learning and hippocampal, neuronal plasticity by repetitive transcranial magnetic stimulation (rTMS). Bioelectromagnetics 2006;27:288-294.

-29 Wang F, Geng X, Tao HY, Cheng Y: The restoration after repetitive transcranial magnetic stimulation treatment on cognitive ability of vascular dementia rats and its impacts on synaptic plasticity in hippocampal CA1 area. J Mol Neurosci 2010;41:145-155.

-30 Zhang N, Xing M, Wang Y, Tao H, Cheng Y: Repetitive transcranial magnetic stimulation enhances spatial learning and synaptic plasticity via the VEGF and BDNF-NMDAR pathways in a rat model of vascular dementia. Neuroscience 2015;311:284-291.

-31 Lenz M, Galanis C, Muller-Dahlhaus F, Opitz A, Wierenga CJ, Szabo G, Ziemann U, Deller T, Funke K, Vlachos A: Repetitive magnetic stimulation induces plasticity of inhibitory synapses. Nat Commun 2016;7:10020.

-32 Lenz M, Platschek S, Priesemann V, Becker D, Willems LM, Ziemann U, Deller T, Muller-Dahlhaus F, Jedlicka P, Vlachos A: Repetitive magnetic stimulation induces plasticity of excitatory postsynapses on proximal dendrites of cultured mouse CA1 pyramidal neurons. Brain Struct Funct 2015;220:3323-3337. 